\title{
Reliable Data Forwarding in Wireless Sensor Networks: Delay and Energy Trade Off
}

\author{
M. K. Chahine ${ }^{1}$, C. Taddia ${ }^{2}$ and G. Mazzini ${ }^{3}$ \\ ${ }^{1}$ Electronics and Communications Department, \\ Mechanical and Electrical Engineering Faculty, University of Damascus \\ 2,3Lepida S.p.A., Bologna \\ ${ }^{1}$ Syria \\ 2,3 Italy
}

\section{Introduction}

Wireless sensor networks (WSNs) are currently the topic of intense academic and industrial studies. Research is mainly devoted to the exploitation of energy saving techniques, able to prolong as much as possible the lifetime of these networks composed of hundreds of battery driven devices[1] [2].

Many envisioned applications for wireless sensor networks require immediate and guaranteed actions; think for example of medical emergency alarm, fire alarm detection, intrusion detection [3]. In such environments data has to be transported in a reliable way and in time through the sensor network towards the sink, a base station that allows the end user to access the data. Thus, besides the energy consumption, that still remains of crucial importance, other metrics such as delay and data reliability become very relevant for the proper functioning of the network [4].

These reasons have led us to investigate a very interesting trade off between the delay required to reliably deliver the data inside a WSN to the sink and the energy consumption necessary to the achievement of this goal.

Typically WSNs consist of many sensor nodes scattered throughout an area of interest that monitor some physical attributes; local information gathered by these nodes has to be forwarded to a sink. Direct communication between any node and the sink could be subject only to just a small delay, if the distance between the source and the destination is short, but it suffers an important energy wasting when the distance increases. Therefore often multihop short range communications through other sensor nodes, acting as intermediate relays, are preferred in order to reduce the energy consumption in the network [5]. In such a scenario it is necessary to define efficient techniques that can ensure reliable communications with very tight delay constraint. In this work we focus our attention on the control of data transport delay and reliability in multihop scenario.

Reliable communications can be achieved thanks to error control strategies: typically the most applied techniques are forward error correction (FEC), automatic repeat request (ARQ) and hybrid FEC-ARQ solutions. A simple implementation of an ARQ is represented by the Stop and Wait technique, that consists in waiting the acknowledgment of each transmitted

Source: Communications and Networking, Book edited by: Jun Peng,

ISBN 978-953-307-114-5, pp. 434, September 2010, Sciyo, Croatia, downloaded from SCIYO.COM 
packet before transmitting the next one, and retransmit the same packet in case it is lost or wrongly received by the destination. The corrupted data can be retransmitted by the source (non cooperative ARQ). Otherwise data retransmissions may be performed by a neighboring node that has successfully overheard the source data transmission (cooperative ARQ) [4].

We have analyzed, in a previous work [6], four reliable data forwarding methods, based on hybrid FEC and non cooperative ARQ techniques, by focusing the attention mainly on their energy consumption. In particular we have compared the direct and multihop communications by defining the regions in which one is more energy efficient than the other, to ensure a predefined reliability of the communication. Furthermore, in case of multihop path, we have defined regions in which the exploitation of FEC hop-by-hop (detect-and-forward solution) can be helpful and energetic efficient with respect to the use of FEC only at the destination (amplify-and-forward solution).

We extend here this analysis by introducing the investigation of the delay required by the reliable data delivery task. To this aim we investigate the delay required by a cooperative ARQ mechanism to correctly deliver a packet through a multihop linear path from a source sensor node to the sink. In particular we analyze the relation between the delay and the coverage range of the nodes in the path, therefore the relation between the delay and the number of cooperative relays included in the forwarding process. This allows to study optimal multihop topologies to improve data forwarding performance in sensor networks while saving energy as much as possible. The cooperative approach is also compared with other non cooperative solutions, and the delay reduction that the cooperative technique allows to obtain with respect to the more trivial non cooperative ones, is shown. We present analytical expressions for the investigated delay in many scenario and we validate them by means of simulation.

Finally a simple simulation analysis of the energy required by the investigated ARQ techniques has been performed, in order to understand the actual trade off shown by the two approaches.

The rest of the work is organized as follows: Section 2 describes the network topology and the ARQ protocols that we have analyzed; Section 3 provides a general mathematical framework to evaluate the average delay required by the proposed ARQ techniques to deliver a correct packet to the sink and closed equations of the delay in some particular topologies; Section 4 introduces a framework to model the energy consumption involved during the data delivery; Section 5 compares the mathematical model results with those obtained with simulations and shows the delays and the energy consumption of different ARQ techniques; Section 6 concludes the chapter.

\section{System model}

Consider a multihop linear path composed by a source node (node $n=1$ ), a sink (node $n=N$ ) and $N-2$ intermediate relay nodes (nodes $n=2, \ldots, N-1$ ), equally spaced, as shown in Figure 1 . The total path is consequently composed by $H=N-1$ subsequent links. Suppose that all the nodes have a circular radio coverage and all the nodes in the path have the same transmission range. Let $R$ be the transmission range of each node, expressed in terms of number of links. This means that whenever a node transmits a packet, due to the broadcast nature of the wireless channel, the packet can be received by a set $S R$ of nodes, 
composed by all the nodes inside the coverage area of the sender that are in a listen state (consider that most of the Media Access Control (MAC) protocols for WSNs are low duty cycle protocols that awake nodes only when necessary, by letting nodes in a sleep state during the rest of the time to save energy [7]).
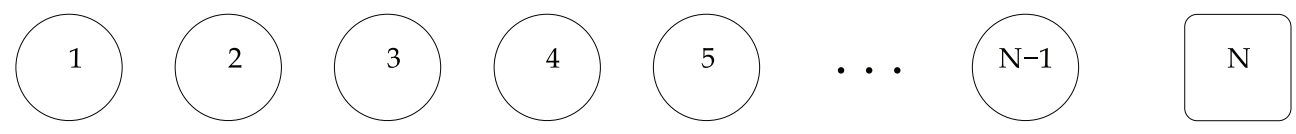

SINK

SOURCE

Fig. 1. Linear multihop path between the source node and the destination sink.

For example, by considering $R=2$ and by referring to Figure 1 , when node 3 broadcasts a packet, the packet can be received by the set $S R$ of nodes, with $S R=\{1,2,4,5\}$. Among the set $S R$ we define the subset $S F$ of the possible forwarders, i.e., the nodes that could forward the data towards the destination. By following the strategy suggested by many geographic routing protocols proposed in literature [9], this subset SF includes only the nodes belonging to $S R$ that have a distance to the destination that is lower than the distance between the transmitter and the destination. By referring to the previous example, $S F$ is composed by nodes 4 and 5 . Generally, for each node $n \in[1, N-1]$ that is transmitting a packet, we can define a set $S F_{n}$ of possible forwarders.

\subsection{Cooperative ARQ}

The cooperative ARQ strategy allows to exploit the collaboration of more relays overhearing the packet transmitted by a node. This approach supposes that for each node $n$, all the nodes belonging to the set $S F_{n}$ are awake and available for the packet reception; the case in which none of them is available will be included as a possible reason of link error packet delivery, as explained in the following mathematical framework (Section 3).

When a node $n$ transmits a packet, the packet is forwarded by the node $n_{F}$ belonging to the set $S F_{n}$, that has correctly received the packet and which is the closest to the destination, in order to complete the data delivery with the minimum number of hops and in the fastest way. Only if no one among the possible forwarder nodes has correctly received the packet, a packet retransmission is requested to the node $n$; otherwise the other nodes of $S F_{n}$ can help the data forwarding process by transmitting the packet, in case they have received it correctly.

Consider, for example, the linear path in Figure 1. The packet delivery process begins from the source node $n=1$, that broadcasts a packet with range $R=2$. In this case the forwarder set is $S F=\{2,3\}$; among these nodes the closest to the destination is $n=3$. If node $n=3$ correctly receives the packet it rebroadcasts it; otherwise if it detects that the received packet is not correct the data delivery will continue from node $n=2$, in case $n=2$ has correctly received the packet; otherwise the process will begin again from the node $n=1$ that proceeds by retransmitting the same packet. This procedure is repeated for all the nodes in the path until a correct packet reaches the destination $n=N$.

\subsection{Non cooperative ARQ}

The non cooperative ARQ strategy defines a transmission range $R$ and schedules communications only between nodes that are $R$ links distant. This means that when a node $n$ 
transmits a packet, all the nodes of $S F_{n}$, except the node distant $R$ link away, can remain in a sleep state, as they do not need to receive the packet, since they will not be involved in the packet forwarding process. In case the packet has not be correctly delivered to the node $n+$ $R$ a retransmission is requested to the sender node $n$.

This ARQ strategy is a generalization of the simple hop-by-hop detect-and-forward technique analyzed in [6], where data packet delivery goes on hop-by-hop baiss and possible retransmissions are required to the previous node of the path; clearly the hop-by hop detect-and-forward case can be derived from the general non cooperative ARQ strategy by choosing $R=1$.

\section{Delay: mathematical framework}

To evaluate the performance of the ARQ strategies discussed above, we define some performance metrics. We are interested in the delay of the packet delivery process, from the source node to the sink, and in the probability distribution of completing the packet delivery in a certain number of steps ( $k$ steps), thus within a certain delay.

By considering that each transmission involves a time slot unit we can proceed by evaluating the delay as multiple unitary time slots and we can calculate it as the number of transmissions needed to deliver a correct packet to the destination. We neglect the delay of ACK or NACK packets. Furthermore when considering wireless communications implicit acknowledgement can also be used [10]: in a multi-hop wireless channel if a node transmits a packet and hears its next-hop neighbor forwarding it, it is an implicit acknowledgement that the packet has been successfully received by its neighbor. The following Subsections $(3.1,3.2,3.3)$ present the Markov chains describing the packet forwarding process and the mathematical framework that calculates the average delay and the delay probability distributions for both the cooperative and non cooperative ARQ strategies.

The validity of this mathematical framework has been verified in the previous work [12] by showing a perfect matching between results obtained by means of simulations with the ones obtained by following the mathematic equations given below.

\subsection{Transition probabilities}

\subsubsection{Cooperative ARQ}

Let $q$ be the probability to successfully deliver a packet to a node inside the transmitter coverage area; $q$ defines the single transmission success probability between two nodes. So $p=1-q$ will be the single transmission error packet probability. For the sake of simplicity the probability $q$ is supposed to be the same inside the coverage area, irrespectively of the distance between the sender and the receiver, provided that they both belong to the subset SF of the sender node. This allows to consider the link error probability not only as a function of the received signal strength, but also dependent on other factors like for example: possible collisions or nodes that are not awake during the packet delivery.

For each node $n$, the probability to correctly deliver a packet to a node that is $R$ links distant (node $n+R$ ) is equal to $q$. So the probability that the packet is not correctly received by this node is $(1-q)$, while it is correctly received from the immediately previous node $(n+R-1)$ with a probability $q$. So with a probability $(1-q) q$ the packet will be forwarded by the node $n+R-1$. If also this node has not correctly received the packet sent by node $n$, event that occurs with a probability $(1-q)^{2}$, with a probability $(1-q)^{2} q$ the packet will be 
forwarded by the node $n+R-2$. If none of the nodes between node $n+1$ and node $n+R$ receives a correct packet it is necessary to ask the retransmission of the packet by the node $n$. It is possible to describe the process concerning one data packet forwarding from the source node $n=1$ to the destination $n=N$ with a discrete time Markov chain. We identify each node in the path with a number $n$, where $n$ varies from 1 (the source) to $N$ (the destination). Each state in the chain represents a node in the path: in particular the process is in state $n$ at a certain time when $n$ is the furthest node, starting from the source, that has correctly received a packet until that time and it has to carry on the forwarding process.

We define $P_{n, n+j}$ as the transition probability between a state $n$ and the state $n+j . P_{n, n+j}$ represents the probability that the data packet broadcasted by node $n$ has been correctly received by node $n+j$ while it has not been correctly received by the other nodes belonging to the subset $S F_{n}$ that are closer to the destination $N$ with respect to the node $n+j$; in other words, $P_{n, n+j}$ is the probability that the next forwarder will be node $n+j$, given that the transmitting node was node $n . P_{n, n+j}$ can be calculated as follows:

- if $1 \leq n \leq N-R$ :

$$
\begin{array}{ll}
P_{n, n+j}=q(1-q)^{R-1} & \text { if } 1 \leq j \leq R \\
P_{n, n+j}=(1-q)^{R} & \text { if } j=0 \\
P_{n, n+j}=0 & \text { otherwise }
\end{array}
$$

- if $N-R+1 \leq n \leq N-1$ :

$$
\begin{array}{ll}
P_{n, n+j}=q(1-q)^{N-n-j} & \text { if } 1 \leq j \leq N-n \\
P_{n, n+j}=(1-q)^{N-n} & \text { if } j=0 \\
P_{n, n+j}=0 & \text { otherwise }
\end{array}
$$

- if $n=N$ :

$$
\begin{array}{ll}
P_{n, n+j}=1 & \text { if } j=0 \\
P_{n, n+j}=0 & \text { otherwise }
\end{array}
$$

Note the there are different $P_{n, n+j}$ equations depending on which state $n$ we are considering. For nodes $n$, with $1 \leq n \leq N-R$, the transition probability from node $n$ to node $n+j$, with $1 \leq j \leq R$, is equal to $q^{\cdot}(1-q)^{R-j}$. In fact, it takes into account that the maximum distance that is possible to cover during a transmission is equal to $R$ links; so if the packet is correctly detected by node $n+R$ we have the transition probability between state $n$ and state $n+R$, with a transition probability $P_{n, n+R}=q$; in case that $i=R-j$ nodes do not correctly receive the packet, there is a transition between state $n$ and state $n+j$, with probability $P_{n, n+j}=$ $q(1-q)^{R-j} ; j$ can varies between 1 and $R$, representing the number of relays belonging to the subset $S F_{n}$. The last $R-1$ nodes that precede the destination node (nodes $n$ with $N-R+1 \leq$ $n \leq N-1$ ) represent an exception, since the distance between the transmitting node and the destination is less than the transmission range of the nodes and therefore in their subsets $S F$ there are less possible cooperative relay nodes. 
An example of Markov chain for a path composed by four nodes $(N=4), H=N-1=3$ links and range $R=2$ is shown in Figure 2, for which we write the transition probability matrix $P_{C}$ as a function of the success link probability.

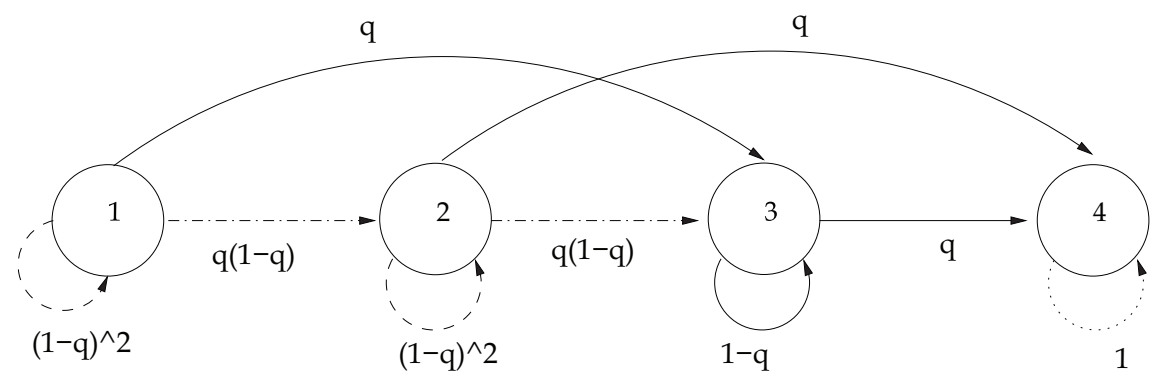

Fig. 2. Markov chain for the topology $N=4, H=3, R=2$.

$$
P_{C}=\left(\begin{array}{cccc}
(1-q)^{2} & (1-q) q & q & 0 \\
0 & (1-q)^{2} & (1-q) q & q \\
0 & 0 & 1-q & q \\
0 & 0 & 0 & 1
\end{array}\right)
$$

The same matrix $P_{C}$ expressed as a function of the error link probability becomes:

$$
P_{C}=\left(\begin{array}{cccc}
p^{2} & (1-p) p & 1-p & 0 \\
0 & p^{2} & (1-p) p & 1-p \\
0 & 0 & p & 1-p \\
0 & 0 & 0 & 1
\end{array}\right)
$$

A similar approach was used in [8] to evaluate the mean number of hops required to realize the Route Request Process by the Ad hoc On-Demand Distance Vector (AODV) routing for ad hoc networks. The approach used here is quite different since it takes into account all the possible retransmissions of the wrong packets.

Note that the Markov chain is characterized by $N-1$ transient states (the source node $n=1$ and all the other relays $n=2,3, \ldots, N-1$ ) and by an absorbing state (the destination sink, node $n=N$, characterized by a transition probability $\left.P_{N, N}=1\right)$. In fact a state $n$ of a Markov chain is defined as transient if a state $i$, with $i \neq n$, exists that is accessible from state $n$ while $n$ is not accessible from $i$; once the system is in state $n$ it can go into one of the states $i=n+j$, with $j \leq \min \{R, N-n\}$ but once the system is in this state $n+j$ it means that the packet has arrived correctly, at least at node $n+j$ therefore node $n$ will not need to retransmit it again; so state $n+j$ is accessible from state $n$ and state $n$ is not accessible from state $n+j$. State $N$ as an absorbing state is a good representation of the physical process that we are analyzing: in fact, this Markov chain describes the packet forwarding process, the travel of a packet from a source towards a destination, where the packet stops and does not have to go in any other place. Results obtained by simulations and presented in the following Section will confirm the correctness of this model. 


\subsubsection{Non Cooperative ARQ}

In case of the non cooperative ARQ the process is composed by a total number of states equal to the ratio $\left\lceil\frac{H}{R}\right\rceil+1$. In fact, as Figure 3 shows, after choosing the range $R$ there are some nodes that will never be involved in the packet forwarding process: for example node 2 in Figure 3 when $R=2$. For each state $n$ of the chain there is a probability $1-p$ that at the next step the packet will be forwarded by the next state of the chain (node $n+\min \{R, N-$ $n\}$ ) and a probability $p$ that it will be retransmitted by the node $n$.
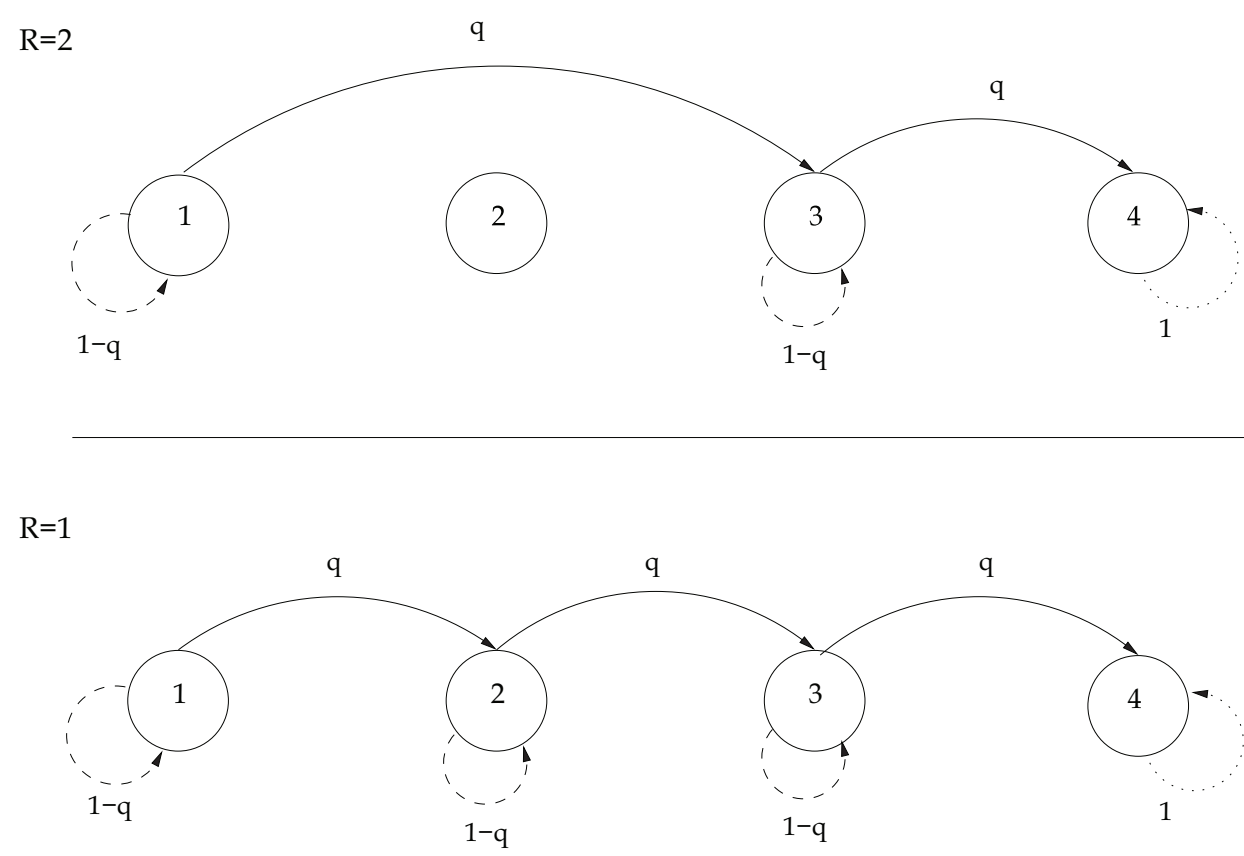

Fig. 3. Markov chain for the topology $N=4, H=3$. Non cooperative ARQ with $R=2$ in the top of the Figure and with $R=1$ in the bottom of the Figure.

The transition probability matrix is a matrix of dimension $\left(\left\lceil\frac{H}{R}\right\rceil+1\right) \times\left(\left\lceil\frac{H}{R}\right\rceil+1\right)$ :

$$
P_{N C}=\left(\begin{array}{cccccc}
p & 1-p & 0 & 0 & \ldots & 0 \\
0 & p & 1-p & 0 & \ldots & 0 \\
0 & 0 & p & 1-p & \ldots & 0 \\
\vdots & \vdots & \vdots & \vdots & \vdots & \vdots \\
0 & 0 & 0 & 0 & \ldots & 1
\end{array}\right)
$$

\subsection{Delay probabilities distribution}

For a generic state $i$ of a discrete time Markov chain [11] described by a generic matrix $P$ of transition probabilities, we define the time of first visit into state i as: $T_{i}=\inf \left\{k \geq 1 \mid X_{k}=i\right\}$, 
where $k$ is the number of visits into the state $i$ and $X_{k}$ is the state in which the system is at time $k$. Generally we denote by $f_{i, j}^{(k)}$ the probability that a system described by a discrete time Markov chain transits for the first time from state $i$ to state $j$ in $k$ steps. This probability is defined as: $f_{i, j}^{(k)}=P\left\{T_{j}=k \mid X_{0}=i\right\}$, where $X_{0}$ is the initial state of the system. ChapmanKolmogorov equations states that the probability $f_{i, j}^{(k)}$ can be calculated as a sum of all the possible combinations of the probabilities of going from state $i$ to state $j$ by going, during the intermediate steps, through the other states of the systems, apart from the state $j$, that has to be reached for the first time at the step $k$. Formally we have:

$$
f_{i, j}^{(k)}=\sum_{s_{1}, s_{2}, \ldots, s_{k-1} \in S \backslash\{j\}} P_{i s_{1}} \cdot P_{s_{1} s_{2}} \cdot \ldots \cdot P_{s_{k-1} j}
$$

where $S$ is the total space of the states and $P_{S_{i} S_{y}}$, (with $i, y \in 1, \ldots, k-1$ ), are the transitions probabilities of the matrix $P$. For each $k \geq 1$ this can be written also as: $f_{i, j}^{(k)}=P_{i, j}^{(k)}-\sum_{i=1}^{k-1} f_{i, j}^{(i)} P_{i, j}^{(k-i)}$. This suggests to calculate the $f_{i, j}^{(k)}$ in a recursive way through the knowledge of the transition probabilities included in the matrix P. For a finite state Markov chain, Equation 4 can be represented in a matrix form: $f_{i, j}^{(k)}$ results to be the element in position $(i, j)$ of the $k$ - th power of the matrix $\tilde{P}$, where $\tilde{P}$ is equal to matrix $P$ except for the $j$ - th row that is taken as a null row in order to remove te possibility of passing through the $j-$ th state in an intermediate step $k^{\prime}<k$.

\subsubsection{Cooperative ARQ}

According to the general definitions given above, we can derive the delay probability distribution in the specific case of the Markov chain described by the matrix $P_{C}$. The probability distribution of ending the process in a certain number $k$ of steps is expressed by the probability that the system transits for the first time from state 1 to state $N$ after $k$ steps. The number of visits for each transient state varies accordingly to the link error probability and to the probability that no one of the relays belonging to the subset $S F_{n}$ of a node $n$ correctly receive the packet and therefore needs to ask for a retransmission of the packet to the sender node $n$. The number of visits to state $N$ is infinite: once the packet arrives at destination the process is ended, it remains into the absorbing state for an infinite time. In fact, in the long term behavior, when time tends to infinity, the steady state probability of state $N$ is one while for all the other transient states $n$ we have $\lim _{k \rightarrow \infty} P_{C_{i, n}}^{(k)}=0, \forall i$, i.e., each state will be absorbed into state $N$. The delay that we are going to evaluate is therefore the mean time of the first visit to state $N$. The Markov chain in fact refers to the delivery of a single packet from the source towards the destination; when considering the transmission of another packet from the source node the process begins again from the state 1 of the Markov chain.

We indicate the probability that the packet is correctly forwarded to the destination in a num- ber of steps $k$ for the cooperative ARQ is defined as:

$$
f_{\mathrm{C}_{1, N}}^{(k)}=\sum_{s_{1}, s_{2}, \ldots, s_{k-1} \in S \backslash\{N\}} P_{1 s_{1}} \cdot P_{s_{1} s_{2}} \cdot \ldots \cdot P_{s_{k-1} N}
$$

This can be easily calculated as the element in position $(1, N)$ of the $k$ - th power of the matrix $\tilde{P}_{C}$, where $\tilde{P}_{C}$ is built equal to matrix $P_{C}$ except for the element $(N, N)$ that is 0 
instead of 1 . These probabilities are a function of the number of hops $H$ composing the path and the range $R$, so it is useful to indicate this dependency by calling these probabilities in the rest of the chapter as $f_{C_{1, N}}^{(k)}(R, H)$. We have found out that for some particular values of the transmission range $(R=1$ and $R=H)$ the probability can be expressed through simple closed form equations. So we have $f_{\mathrm{C}_{1, N}}^{(k)}(1, H)=\left(\begin{array}{c}k-1 \\ H-1\end{array}\right) p^{k-H}(1-p)^{H} \quad$ and $f_{C_{1, N}}^{(k)}(H, H)=p^{k}(1-p)$.

\subsubsection{Non cooperative ARQ}

The probability $f_{C_{1, N}}^{(k)}(R, H)$ can be calculated by following the general approach described at the beginning of subsection 3.2 applied to the matrix $P_{N C}$. Note that $f_{C_{1, N}}^{(k)}(R, H)$ results to be described by the following closed equation:

$$
f_{N C_{1, N}}^{(k)}(R, H)=\left(\begin{array}{c}
k-1 \\
\left\lceil\frac{H}{R}\right\rceil-1
\end{array}\right) p^{k\left\lceil\left\lceil\frac{H}{R}\right\rceil\right.}(1-p)^{\left\lceil\frac{H}{R}\right\rceil}
$$

\subsection{Average delay}

The average delay is represented by the absorption time into last state of the chain starting from the source. The mean time of first visit from state $i$ to state $j$ of a discrete time Markov chain, called $T_{i, j}$ is defined as follows:

$$
T_{i, j}=\left\{\begin{array}{cc}
\infty & \text { if } \sum_{k=1}^{\infty} k f_{i, j}^{(k)}<1 \\
\sum_{k=1}^{\infty} k f_{i, j}^{(k)} & \text { if } \sum_{k=1}^{\infty} k f_{i, j}^{(k)}=1
\end{array}\right.
$$

When $\sum_{k=1}^{\infty} f_{i, j}^{(k)}=1$ the time $T_{i, j}$ is univocally solution of the following equation:

$$
T_{i, j}=1+\sum_{s \neq j} P_{i, s} T_{s, j}
$$

By fixing an arrival state $j$, equation 7 allows to obtain a linear system whose solutions are the mean time of first transition from each one of the possible initial states $i,(\forall i \in S \backslash\{j\}$, where $S$ is the total space of the states), to the final state $j$.

\subsubsection{Cooperative ARQ}

According to the general definitions given above, we can derive the average delay in the specific case of the Markov chain described by the matrix $P_{C}$. The delay we want to evaluate is the absorption time to state $N$ by starting from state 1, i.e., the mean time of first visit from state 1 to state $N$. Since in our case the state $N$ is an absorbing state the condition $\sum_{k=1}^{\infty} f_{1, N}^{(k)}=1$ is verified; in fact the probability for each transient state to be absorbed into 
state $N$ is equal to one. So we can calculate the mean time $T_{C_{1, N}}$ by solving the linear system defined in Equation 7, where the transition probabilities $P_{i, s}$ are taken from the matrix $P_{C}$ :

$$
T_{C_{i, N}}=1+\sum_{s \neq N} P_{i, s} T_{C_{s, N}}
$$

where $i=1,2, \ldots, N-1$.

Since it is a function of the number of links $H$ composing the path and of the range $R$, in the rest of the chapter the term $T_{C}(R, H)$ refers to that quantity. We omit the indexes $1, N$ defining the starting and the final node, for the sake of simplicity, since they nevertheless are always the source node 1 and the destination $N$. We have analyzed the possibility to express the delay in a closed form, for each value of the total number of links composing the path, $H$, and for some particular values of the transmission range: $R=1, R=H$, $R=H-1$ and $R=H-2$. When $R=1$ the delay has the following expression:

$$
T_{C}(1, H)=H /(1-p)
$$

When $R=H$ we have:

$$
T_{C}(H, H)=1 /(1-p)
$$

When $R=H-1$ we have found the following Equation:

$$
\begin{aligned}
T_{C}(H-1, H) & =\frac{2+\sum_{i=1}^{H-2} p^{i}}{(1-p) \sum_{i=1}^{H-2} p^{i}} \\
& =\frac{1}{1-p}+\frac{p}{p-p^{H}}
\end{aligned}
$$

When $R=H-2$ the following expression is valid:

$$
\begin{aligned}
T_{C}(H-2, H)= & \frac{1}{(1-p)\left[\sum_{i=1}^{H-3} p^{i}\right]^{2}} \cdot \\
& \cdot\left[\sum_{i=-1}^{H-5}(3+i) p^{i+1}+\right. \\
& \left.+H p^{H-3}+\sum_{i=0}^{H-4} p^{H-2+i}(H-3-i)\right] \\
& =\frac{p^{4}(2-p)+p^{2 H}+p^{H+1}\left[1-5 p+2 p^{2}\right]}{(1-p)\left[p^{2}-p^{H}\right]^{2}}
\end{aligned}
$$




\subsubsection{Non cooperative ARQ}

The average delay required by the non cooperative approach can be derived by following the general approach described above and applied to the matrix $P_{N C}$. It can also be derived by simply thinking that is the product between the mean number of hops in which the total path is divided once the transmission range $R$ has been chosen, (that turns to be $\left\lceil\frac{H}{R}\right\rceil$ ), and the mean number of transmission needed to correctly deliver a packet between two nodes $R$ links distant. Suppose that $p$ is the link error probability at distance $R$. We call $P_{a}$ the probability to make $a$ attempts in order to deliver a correct packet in a single hop communication; $P_{a}$ can be calculated as the probability to make one successful transmission (event that happen with a probability $1-p$ ) and $a-1$ failures (event happening with probability $\left.p^{a-1}\right)$. The mean number of transmissions $E[t x]$ needed per single hop is derived as follows:

$$
\begin{aligned}
E[t x] & =\sum_{a=1}^{\infty} a P_{a} \\
& =\sum_{a=1}^{\infty} a p^{a-1}(1-p)=\frac{1}{1-p}
\end{aligned}
$$

The delay is therefore calculated as:

$$
T_{N C}(R, H)=\frac{\left\lceil\frac{H}{R}\right\rceil}{1-p}
$$

\section{Energy model}

In order to better evaluate the performance of the proposed ARQ strategies, we also investigate the energy consumption required by them in different scenarios. This allows to obtain useful trade offs between energy consumption and delay requested to accomplish a task.

We define a simple energetic model, by referring to the considerations made in [6]. Suppose having a scenario with $H$ hops between the source and the destination and having fixed distance between two subsequent nodes.

In more detail, the energy $E$ required in a point-to-point communication between two nodes is the sum of two contributions: the energy spent by the transmitter for transmitting a packet, $E_{T X}$, and the energy spent by the node receiving the data packet, indicated with $E_{R X}$. More in detail the energy $E_{T X}=E_{c}+E_{d}(R)$ comprises two contributes: the energy spent by the transceiver electronics and by the processor to encode the packet with a preselected FEC code to reveal the errors in the packet, $E_{c}$ and a contribution $E_{d}(R)$ proportional to the distance between the nodes involved in the communication and the signal to noise ratio desired at the destination. The energy $E_{R X}$ comprises the energy of the transceiver electronics and the energy spent by the processor in decoding the packet, $E_{c}$. The total energy required to deliver a correct packet to the destination, $E_{T O T}$, can be calculated as the energy spent for a transmission multiplied by the total number of transmissions performed during the 
forwarding process, $N_{T X}$, added to the energy spent for a reception multiplied by $N_{T R}$, the total number of receptions occurred during the forwarding process: $E_{T O T}=N_{T X} E_{T X}+N_{R X} E_{R X}$. Let $\alpha$ be the ratio between $E_{c}$ and the term $E_{d}(1)$, that is the contribution of energy $E_{d}$ required to send a packet to a node that is 1 link distant from the sender: $\alpha=E_{c} / E_{d}(1)$. We proceed by normalizing the total energy with respect to the contribute $E_{d}(1)$. Therefore the normalized energy $\hat{E}_{T O T}(R, H)$ for a path composed by $H$ links and with a transmission range $R$ is:

$$
\hat{E}_{T O T}(R, H)=\left(R^{\eta}+\alpha\right) N_{T X}+\alpha N_{R X}
$$

where $N_{T X}$ and $N_{R X}$ refers to the specific total number of transmissions and receptions of the ARQ strategy under analysis and $\eta$ is the path loss exponent.

\section{Numerical results: delay-energy trade off}

Results related to the performance in terms of delay and energy consumption of the two mentioned ARQ approaches and their correlations and dependencies with various parameters, such as the communication range $R$ and the sensor node circuitry (with the parameter $\alpha$ ) has been deeply investigated and presented in the previous work [12].

In this Section we rather show the performance of the proposed cooperative and non cooperative ARQ strategies in terms of delay and energy consumption, by pointing up the trade off between these two important metrics.

In order to monitor also the comparison between the two ARQ approaches, we investigate in our trade off study the ratio between the results obtained with the cooperative solution and the non cooperative one, for both two metrics, delay and energy consumption.

The results presented in this section have been tested by means of simulations by following the energetic model described in Section 4. Let us precise that the results presented in the following have been obtained only by means of simulation, since it is not trivial to derive a precise mathematical model to calculate the number $N_{R X}$ for the cooperative ARQ. In fact the number of nodes receiving the packet or each packet transmissions depends on the node that is transmitting: by referring to the matrix $P_{C}$, it depends on the state $n$ of the sender node: the number of receiving nodes for each packet transmission is $R$ if $1 \leq n \leq N-R$, but it is less than $R$ for the states $n$ of the chain that are $N-R+1 \leq n \leq N-1$.

Figures 4 and 5 shows the tradeoff between the delay and the energy consumption. As an example a path composed by $H=10$ hops has been considered. The communication range of the nodes has been taken equal to $R=3$ or $R=5$ and different values of the parameter $\alpha=5$, 15,30 has been tested. In order to compare the different ARQ mechanisms in a realistic scenario, we have estimated the range of values of the parameter a by referring to an actual sensor node, the $\mu$ AMPS1, as followed in [6]. We observe that for these specific hardware constraints the parameter $\alpha$ can vary in a range between 1 and 50. We have used values of $\alpha$ between these boundaries to compare the energy spent by the different ARQ strategies. Accordingly to the scenario parameters $(R, \alpha)$ and as a function of the channel quality $P$ this graph allows to easy calculate the gain achievable in terms of energy and latency by choosing one of the two proposed ARQ approaches.

Accordingly to the scenario parameters $(R, \alpha)$ and as a function of the channel quality $P$ this graph allows to easy calculate the gain achievable in terms of energy and latency by choosing one of the two proposed ARQ approaches. 
Figure 4 shows as $x$-axis the ratio between the delay of the cooperative ARQ technique and the delay of the non cooperative one and as y-axis the ratio between the energy consumption required by the cooperative approach and the non cooperative one. In this graph the cooperative and non cooperative techniques have been implemented with the same communication range for each node. While in Figure 5 the comparison concerns the cooperative ARQ with a generic range $R$ and the non cooperative solution implemented with communication range $R=1$ (hop-by-hop detect-and-forward case). In both the Figures, results are plotted for different values of the link error probability $p$, varying between 0.1 and 0.9 , as indicated in the graphs.

Figure 4 evidences that while the delay required by the cooperative solution is always less than the non cooperative one, a trade off is present concerning the energy consumption, that

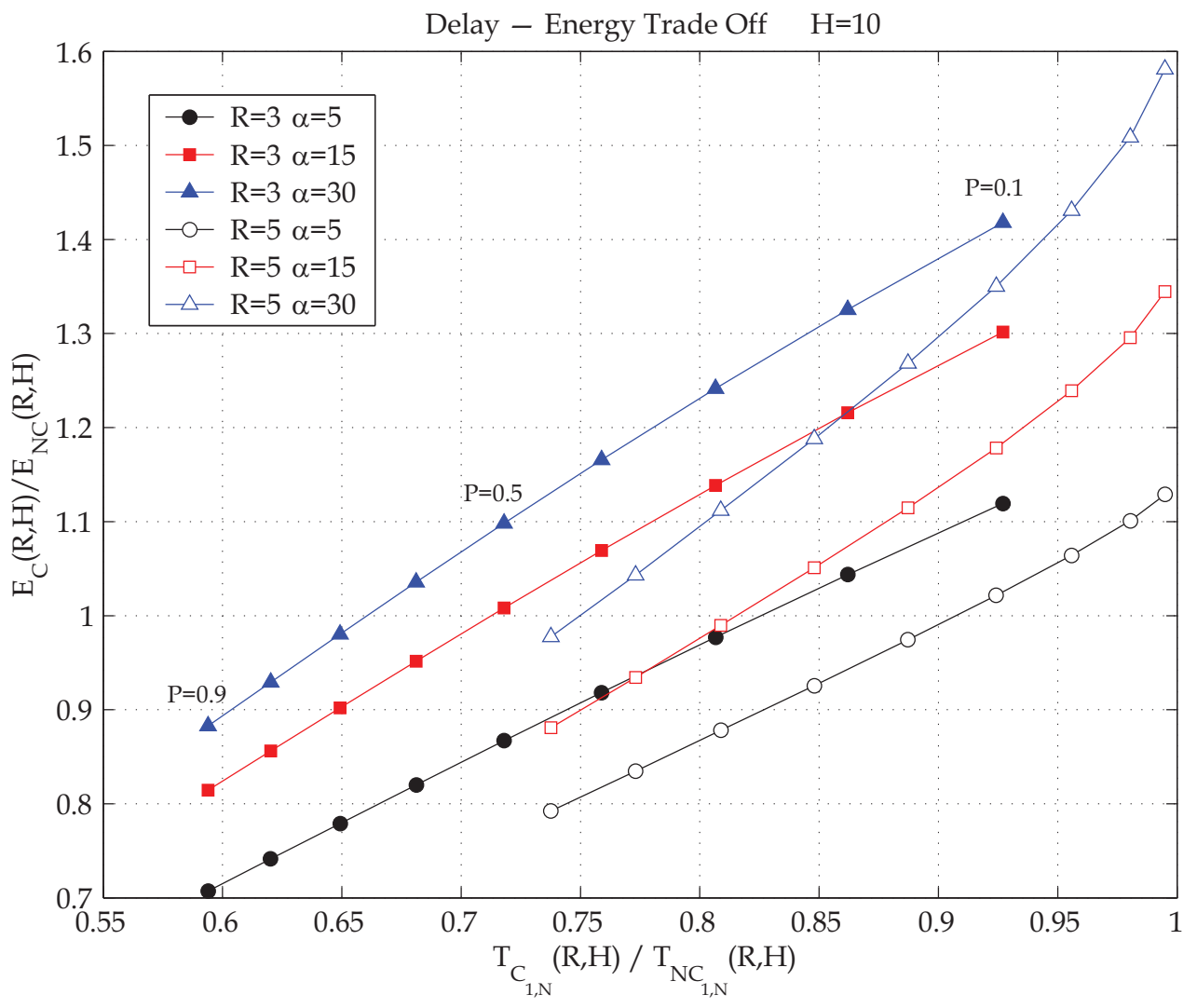

Fig. 4. Delay-Energy tradeoff. Comparison between the cooperative and the non cooperative ARQ techniques both with the same communication range $R$ for the nodes. The path is composed by $H=10$ links. 
depends on the ratio $\alpha$, on the packet error probability per link $p$ and on the range $R$. In particular, we can see that the cooperative ARQ turns out to be an energetic efficient solution with respect to the non cooperative ARQ when the link reliability is quite low and when the ratio $\alpha$ is sufficiently low. Performance in terms of delay reduction are even bigger if comparing the cooperative ARQ (with range $R$ ) with the non cooperative single hop detect- and-forward $(R=1)$, as evidenced in Figure 5. Also in this case a trade off between delay and energy can be achieved: notice that there are regions of $p$ and $\alpha$ (when $\alpha$ is sufficiently low in this case) for which the cooperative ARQ, besides giving better delay performance, also can help in saving the nodes energy and thus extending the network lifetime.

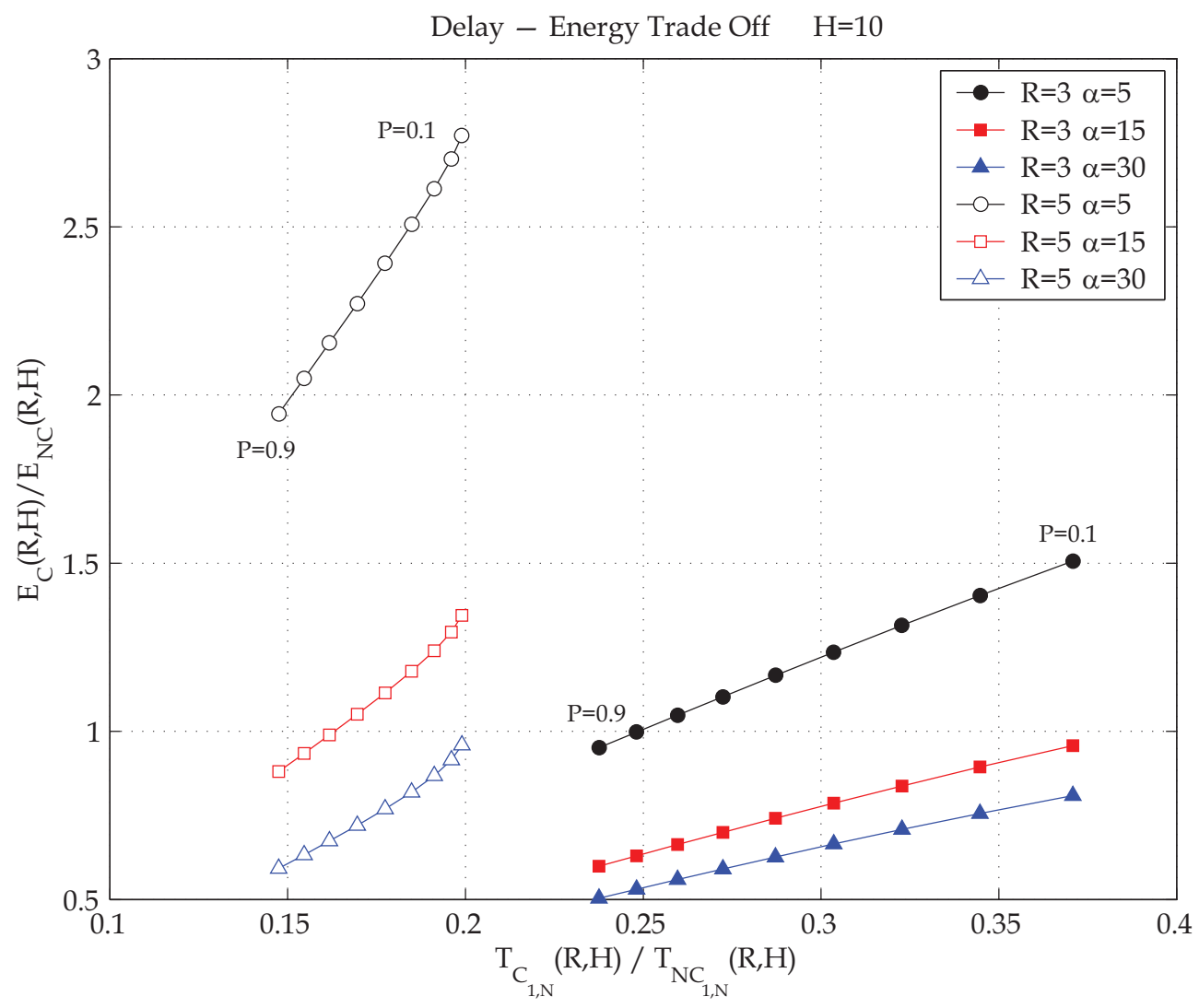

Fig. 5. Delay-Energy tradeoff. Comparison between the cooperative ARQ technique with communication range $R$ and the non cooperative ARQ single hop decode-and-forward approach (with $R=1$ ). The path is composed by $H=10$ links. 


\section{Conclusions}

This work has deeply presented an important trade off between energy consumption and delay in the task of reliable data delivery between a source node and a destination sink in a wireless sensor network. We have presented the performance in terms of delay and energy consumption of cooperative and non cooperative ARQ techniques that allows to ensure reliable communications in WSNs for delay constraints applications. Our investigations have showed that the proposed cooperative ARQ is a successful technique. In particular the cooperative solution, besides showing always better performance concerning the timeliness of data delivery, with respect to the non cooperative approach, can in some scenario outperform the trivial non cooperative hop-by-hop detect and forward technique also in terms of energy saving.

\section{References}

[1] W.Ye, J. Heidemann, D. Estrin, "An energy-efficient MAC protocol for wireless sensor networks", Infocom 2002, 23-27 June, pp. 1567 - 1576, vol.3.

[2] V. Raghunathan, C. Schurgers, Sung Park, M. Srivastava, "Energy-aware wireless microsensor network", IEEE Signal Processing Magazien, March 2002, pp.40-40, vol.19.

[3] L. Bernardo, R. Oliveira, R. Tiago, P. Pinto, "A Fire Monitoring Application For Scattered Wireless Sensor Networks". WinSys 2007, 28-31 July, Barcelona.

[4] I. Cerutti, A. Fumagalli, P.Gupta, "Delay Models of Single-Source Single-Relay Cooperative ARQ Protocols in Slotted Radio Networks with Poisson Frame Arrivals", Infocom 2007, pp. 2276-2280, vol.16

[5] Z. Shelby, C. Pomalaza-Raez, J. Haapola, "Energy optimization in multihop wireless embedded and sensor networks", PIMRC 2004, 5-8 Sept, pp. 221-225, vol.1.

[6] C. Taddia, G. Mazzini, "On the Energy Impact of Four Information Delivery Methods in Wireless Sensor Networks",IEEE Communication Letters, Feb. 2005, Vol. 9, n. 2, pp. 118-120.

[7] S. Ramakrishnan, H. Huang, M. Balakrishnan, J. Mullen, "Impact of sleep in a wireless sensor MAC protocol", VTC Fall 2004, 26-29 Sept, pp.4621-4624, vol. 7.

[8] C.Taddia, G.Mazzini, "An Analytical Model of the Route Acquisition Process in AODV Protocol", IEEEWirelessCom 2005, 13-16 June, Hawaii.

[9] M. Zorzi, R. Rao, "Geographic random forwarding (GeRaF) for ad hoc and sensor networks: multihop performance", IEEE Transaction on Mobile Computing, pp. 337348, vol.2, issue 4, 2003.

[10] T. Hwee-Pink, K.G. Winston, L. Doyle, "A Multi-hop ARQ Protocol for Underwater Acoustic Networks", Proceedings of the IEEE/OES OCEANS Conference, 18-21 June 2007, Aberdeen, Scotland.

[11] S. Karlin, H.M. Taylor, "A First Course in Stochastic Processes", Academic Press. 
[12] C. Taddia, G.Mazzini, M.K.Chahine, K. Shahin, "Reliable Data Forwarding for Delay Constraint Wireless Sensor Netwrorks", International Conference on Information and Communication Technologies, ICTTA 2008, 7-11 April, Damascus, Syria. 


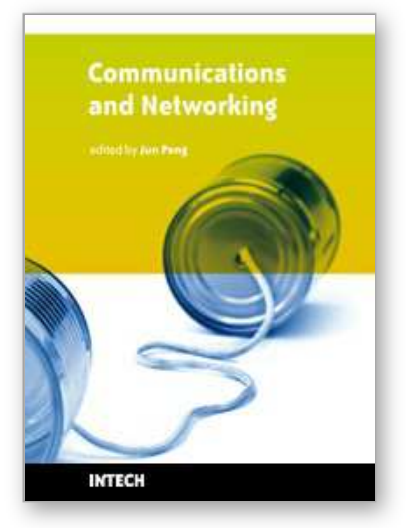

\section{Communications and Networking \\ Edited by Jun Peng}

ISBN 978-953-307-114-5

Hard cover, 434 pages

Publisher Sciyo

Published online 28, September, 2010

Published in print edition September, 2010

This book "Communications and Networking" focuses on the issues at the lowest two layers of communications and networking and provides recent research results on some of these issues. In particular, it first introduces recent research results on many important issues at the physical layer and data link layer of communications and networking and then briefly shows some results on some other important topics such as security and the application of wireless networks. In summary, this book covers a wide range of interesting topics of communications and networking. The introductions, data, and references in this book will help the readers know more abut this topic and help them explore this exciting and fast-evolving field.

\section{How to reference}

In order to correctly reference this scholarly work, feel free to copy and paste the following:

Gianluca Mazzini, Mohamed Khaled Chahine and Chiara Taddia (2010). Reliable Data Forwarding in Wireless Sensor Networks: Delay and Energy Trade Off, Communications and Networking, Jun Peng (Ed.), ISBN: 978953-307-114-5, InTech, Available from: http://www.intechopen.com/books/communications-andnetworking/reliable-data-forwarding-in-wireless-sensor-networks-delay-and-energy-trade-off

\section{INTECH}

open science | open minds

\section{InTech Europe}

University Campus STeP Ri

Slavka Krautzeka 83/A

51000 Rijeka, Croatia

Phone: +385 (51) 770447

Fax: +385 (51) 686166

www.intechopen.com

\section{InTech China}

Unit 405, Office Block, Hotel Equatorial Shanghai

No.65, Yan An Road (West), Shanghai, 200040, China

中国上海市延安西路65号上海国际贵都大饭店办公楼 405 单元

Phone: +86-21-62489820

Fax: $+86-21-62489821$ 
(C) 2010 The Author(s). Licensee IntechOpen. This chapter is distributed under the terms of the Creative Commons Attribution-NonCommercialShareAlike-3.0 License, which permits use, distribution and reproduction for non-commercial purposes, provided the original is properly cited and derivative works building on this content are distributed under the same license. 